\title{
Gut Microbiota: An Integral Moderator in Health and Disease
}

\author{
Qingqing Feng ${ }^{1}$, Wei-Dong Chen ${ }^{2,3 *}$ and Yan-Dong Wang ${ }^{1 *}$ \\ ' State Key Laboratory of Chemical Resource Engineering, College of Life Science and Technology, Beijing University of \\ Chemical Technology, Beijing, China, ${ }^{2}$ Key Laboratory of Receptors-Mediated Gene Regulation and Drug Discovery, School \\ of Medicine, Henan University, Kaifeng, China, ${ }^{3}$ Key Laboratory of Molecular Pathology, School of Basic Medical Science, \\ Inner Mongolia Medical University, Hohhot, China
}

\section{OPEN ACCESS}

Edited by: Miguel A. Aon,

National Institute on Aging (NIH),

United States

Reviewed by:

Roberto Mazzoli,

Università degli Studi di Torino, Italy

Allison Clark,

Open University of Catalonia, Spain

*Correspondence:

Yan-Dong Wang

ydwangbuct2009@163.com

Wei-Dong Chen

wdchen666@163.com

Specialty section:

This article was submitted to

Microbial Physiology and Metabolism,

a section of the journal

Frontiers in Microbiology

Received: 19 September 2017

Accepted: 23 January 2018

Published: 21 February 2018

Citation:

Feng $Q$, Chen W-D and Wang Y-D (2018) Gut Microbiota: An Integral

Moderator in Health and Disease.

Front. Microbiol. 9:151.

doi: 10.3389/fmicb.2018.00151
The gut microbiota, as the main member in gut microecology, is an essential mediator in health and disease. The gut microbiota interacts with various organs and systems in the body, including brain, lung, liver, bone, cardiovascular system, and others. Microbiotaderived metabolites such as the short chain fatty acid (SCFA) butyrate are primary signals, which link the gut microbiota and physiology. Recently, the gut microbiota has been identified as the origin of a number of diseases by influencing the related cell signaling pathways such as WNT/beta-catenin pathway in colorectal cancer and $T$ cell receptor signaling in the central nervous system. Moreover, several microRNAs participate in signaling networks through the intervention of the gut microbiota. The interaction between the gut microbiota and miRNAs plays a crucial role in vascular dysfunction and hepatocellular carcinoma (HCC). In this review, we will report and discuss recent findings about the crosstalk between the gut microbiota and physical organs and how the gut microbiota and miRNAs regulate each other while influencing the host via genes, proteins, or metabolites.

Keywords: gut microbiota, miRNA, intestinal disease, liver disease, lung

\section{INTRODUCTION}

Growing investigations on host-microbe interactions have revealed that the gut microbiota is a critical mediator in maintaining health (Holmes et al., 2011). The "gut microbiota" generally refers to the diverse microbial community (bacteria, fungi, archaea, viruses, and protozoa) that colonizes their host's gastrointestinal tract (Shukla et al., 2017). The composition of gut microbiome is similar at the phylum level (mainly Bacteroidetes and Firmicutes), but diversity and richness of species is variable between individuals (Tremaroli and Backhed, 2012). Host genetics, environmental factors, diet, disease, stress, and some other factors decide the structure of the gut microbiota (Shukla et al., 2017) while the microbiota dictates host's health and diseases via genes, proteins or metabolites (Van de Wiele et al., 2016).

Physiological energy homeostasis could be regulated by microbiota. For example, Donohoe et al. (2011) reported that microbiota-produced butyrate could provide energy to colonocytes and then prevent autophagy in the colon. In addition, physiological homeostasis may be disrupted by the microbiota, resulting in disruption of host metabolism, immune dysregulation, neurological and cognitive dysfunction and others (Roy and Trinchieri, 2017). This disrupted status would cause a series of disorders, including obesity, diabetes, autoimmunity, allergy, inflammatory bowel disease (IBD) and cancer (Holmes et al., 2011; Buret, 2016). Emerging data of the gut microbiota reveal that the microbiota is involved in diverse diseases via gut-brain axis (Baruch and Schwartz, 2016; 
Mu et al., 2016; Dinan and Cryan, 2017; Kennedy et al., 2017), gut-lung axis (Budden et al., 2017; Shukla et al., 2017), gut-liver axis (Nicolas et al., 2017; Xue et al., 2017), gut-bone axis (Ohlsson and Sjogren, 2015; Yan et al., 2016; Ridler, 2017; Villa et al., 2017), gut-vascular axis (Zhu et al., 2016; Li et al., 2017), and other axes.

MicroRNAs (miRNAs) are regarded as regulators of various diseases (Rupaimoole and Slack, 2017). It has been found that microbiota-produced butyrate regulated hepatic cell apoptosis and proliferation by inducing miR-22 expression (Pant et al., 2017). The research about gut microbiota-miRNA interaction has revealed that the gut microbiota could be regulated by hostsecreted miRNAs and the gut microbiota may affect the host via inducing miRNAs (Liu et al., 2016; Belcheva, 2017). The gut microbiota-miRNAs-diseases axis could serve as a new direction for future investigation.

In this review, we summarize the physiological and pathological functions of the gut microbiota including signals from gut to other organs. Specifically, we highlight the new functions about the gut microbiota in diseases and how the gut microbiota monitors the host status by governing special miRNAs.

\section{MICROBE-HOST COMMUNICATION IN DISEASE}

Since the gut microbiota settles in gastrointestinal tract, it is unsurprising that microbiota contributes to regulating intestinal diseases (Holmes et al., 2011). However, emerging evidence indicates that the microbiota is implicated in extraintestinal organs due to the difference of microbiota composition. This altered microbiota composition might be attributed to diet or the environment (cold, antibiotics, use of probiotics, and the occurrence of infections) and dominate the host's physiological state. More substantially, communication pathways between the gut and other organs are mediated by direct neuronal contact, enteroendocrine cells, immune cells and microbial metabolites (Schroeder and Backhed, 2016). Part of cell signaling pathways in the process has been presented in Table 1. It is pivotal to explore how the microbiota communicates with host and develops disease. These relevant investigations will be revealed as follows.

\section{Gut Microbiota and Intestinal Disease}

The gastrointestinal tract is an extremely complex organ system. In symptom, dysbiosis of the gut microbiome forms part of the etiology of various gastrointestinal diseases, especially colorectal cancer (CRC) and IBD (O'Keefe et al., 2009; Zhu et al., 2011; Ohigashi et al., 2013; Yang et al., 2013; Gao et al., 2015; Yuan et al., unpublished). De Filippo et al. (2010) investigated the gut microbial composition from European children (a modern western diet) and Africa children (a rural diet with high fiber) and found a significant abundance of Bacteroidetes and a significant decrease of Firmicutes in Africa children compared with European children. In parallel, Enterobacteriaceae (Shigella and Escherichia) were higher in European children than that in Africa children (De Filippo et al., 2010). Nucleotidebinding oligomerization domain-containing protein 2 (one of the genes associated with IBD) risk allele count increases with Enterobacteriaceae relative abundance, which may well explain the higher occurrence of IBD in Europe than that in Africa (Farrokhyar et al., 2001; Knights et al., 2014). Intestinal microbial composition is also a common factor in CRC. Gao et al. (2015) reported that the levels of Firmicutes and Fusobacteria were higher, while the abundance of Proteobacteria was decreased in CRC patients (Gao et al., 2015). Members of Fusobacteria has been identified as pro-inflammatory via recruiting myeloidderived tumor-infiltrating immune cells such as tumor-associated macrophages (TAMs), dendritic cells (DCs), and myeloidderived suppressor cells (MDSCs) (Kostic et al., 2013; Patel et al., 2016).

In addition, the gut microbiota affects the host via the immune system or metabolites. Short chain fatty acid (SCFA) butyrate, as a histone deacetylase (HDAC) inhibitor, is the most commonly studied metabolite. Ohigashi et al. (2013) reported that SCFAs (acetic acid, propionic acid, and butyric acid) were significantly decreased in CRC group (Ohigashi et al., 2013). Numerous studies also confirmed butyrate is involved in several signaling pathways in CRC (Table 1), including suppressing nuclear factorkappa B (NF-kB), inducing WNT/beta-catenin activity and apoptosis, activating mitogen-activated protein kinase (MAPK) signaling pathway by upregulation of GADD153 or activation of phosphorylation of c-jun N-terminal kinase (JNK) (Scott et al., 2008; O’Keefe et al., 2009; Zhang et al., 2010; Fung et al., 2012; Yang et al., 2013; Zeng et al., 2014). In addition, lipoteichoic acid (LTA) produced by the gut microbiota, a TLR2 ligand, promoted inflammation in host. However, LTAdeficient Lactobacillus acidophilus inhibited inflammation and could prevent against colon cancer, colitis and polyposis (Yang et al., 2013). Overall, paying attention to the roles that the gut microbiota plays in regulating immune responses and tumorigenesis in the gastrointestinal tract will be necessary for disease prevention.

\section{Microbiota-Gut-Brain Axis in CNS}

Adaptive immunity (especially $\mathrm{T}$ cells) and innate immune system contribute to gut-brain communications via regulation of immune activity and the production of proinflammatory cytokines in IBD, irritable bowel syndrome (IBS) and functional dyspepsia (Leue et al., 2017; Powell et al., 2017). Alzheimer's disease $(\mathrm{AD})$ is a form of dementia associated with aging, and signaling pathways of gut-brain axis participate in the disease. The microbiota composition changes due to aging and environmental factors, while function of the intestinal mucosal barrier reduces, and bacterial amyloids and lipopolysaccharides (LPSs) systemically leak with increasing age. These toxins are translocated to the CNS due to the increase of blood-brain barrier permeability associated with aging. At the same time, gut dysbiosis affects amyloid beta peptide physiology possibly by changing energy metabolism and insulin resistance. LPS/amyloids increases microglial activation through TLRs-mediated inflammatory response, induces inflammation, and reduces the phagocytic clearance 
TABLE 1 | Gut microbiota influences cell signaling pathways in diseases.

\begin{tabular}{|c|c|c|c|}
\hline Disease & $\begin{array}{l}\text { Signal molecular derived } \\
\text { from gut microbiota }\end{array}$ & Cell signaling pathways & Reference \\
\hline \multirow[t]{2}{*}{ Colorectal cancer } & Butyrate & NF-кB MAPK Wnt/ß-catenin & $\begin{array}{l}\text { Stempelj et al., 2007; Scott et al., 2008; } \\
\text { O'Keefe et al., 2009; Zhang et al., 2010; } \\
\text { Fung et al., 2012; Yang et al., 2013; Zeng et al., } \\
2014\end{array}$ \\
\hline & LPS & T cell receptor signaling ERK1/2 & Zhu et al., 2011 \\
\hline \multirow{3}{*}{ CNS } & SCFAs & $\begin{array}{l}\text { Dopamine signaling in Parkinson's } \\
\text { disease }\end{array}$ & Sampson et al., 2016 \\
\hline & Amyloids and LPS & $\begin{array}{l}\text { Amyloid plaque and neurofibrillary } \\
\text { tangle formation in Alzheimer's disease }\end{array}$ & Köhler et al., 2016 \\
\hline & $\begin{array}{l}\text { Dopamine, serotonin }(5-\mathrm{HT}) \text {, } \\
\text { neuropeptide, substance and } \\
\text { vasoactive intestinal peptide }\end{array}$ & T cell receptor signaling & Powell et al., 2017 \\
\hline Liver disease & Butyrate & $\begin{array}{l}\text { PI3K/Akt signaling Mitochondrial } \\
\text { control of apoptosis }\end{array}$ & Pant et al., 2017 \\
\hline \multirow[t]{2}{*}{ Lung disease } & LPS SCFA & T cell receptor signaling & $\begin{array}{l}\text { Trompette et al., 2014; Thorburn et al., 2015; } \\
\text { Budden et al., 2017; Gray J. et al., 2017; } \\
\text { Tamburini and Clemente, } 2017\end{array}$ \\
\hline & Polysaccharide A & & \\
\hline \multirow[t]{2}{*}{ Vascular dysfunction } & LPC18:1 & AKT & Li et al., 2017 \\
\hline & SCFAs & MCP-1/CCR-2 signaling IL-17 signaling & Karbach et al., 2016 \\
\hline \multirow[t]{2}{*}{ Osteoporosis } & & $\mathrm{PI} 3 \mathrm{~K} / \mathrm{AKT} / \mathrm{mTOR}$ & Bakker et al., 2016 \\
\hline & SCFAs & T cell receptor signaling & Ohlsson and Sjogren, 2015 \\
\hline
\end{tabular}

ND, no data available.

of amyloid. Subsequent inflammation would lead to the onset/progression of neurodegeneration and amyloid beta peptide accumulation (Köhler et al., 2016). Moreover, in a model of Parkinson's disease, the gut microbiota could regulate motor deficits and neuroinflammation via SCFAs. SCFAs modulate $\alpha$-synuclein-induced microglia activation in the brain and promote $\alpha$-synuclein-dependent neuroinflammation and motor dysfunction (Sampson et al., 2016) (Table 1). Microbial metabolites produced by an imbalanced microbiota aggravated and participated in the pathogenesis of certain neurologic conditions. Accurate identification of relevant flora and metabolites may benefit from the discovery of new drug targets. Currently, very limited data about the interactions of the microbiota-gut-brain from human studies exist. The roles of the microbiota-gut-brain axis in CNS are highly plausible. New animal and clinical studies may provide novel approaches for prevention and treatment of mental illness (Fung et al., 2017).

\section{Gut Microbiota and Liver Disease}

The gut microbiota may modulate alcohol liver disease (ALD), non-alcoholic fatty liver disease (NAFLD), cirrhosis, and even hepatic carcinoma (Betrapally et al., 2017). NAFLD has been the extremely frequent origin of chronic liver disease with growing obesity. Probiotics participate in obstructing the development of NAFLD via suppression of the LPS-TLR4 signaling pathway
(Xue et al., 2017). Hepatic gluconeogenesis could be controlled by the gut microbiota. Probiotics induced hepatic gluconeogenesis while caecal microbiota from obesity reduced markers of hepatic gluconeogenesis (Nicolas et al., 2017). Recent report showed the mechanistic link between microbiota and hepatocellular carcinogenesis (Xie et al., 2017). The report revealed that sexbased disparity in liver carcinogenesis is associated with the gut microbiota, bile acids, and tumor-suppressive microRNAs (miR26a, miR-26a-1, miR-192, miR-122, miR-22, and miR-125b) in male and female mice treated with the streptozotocin-high fat diet (STZ-HFD). Microbiota regulated bile acids and microRNAs promoting the hepatocellular carcinoma (HCC) in a male mouse model, however, the regulatory mechanism is unclear. Elevated levels of farnesoid X receptor (FXR), a bile acid nuclear receptor, in female mice may increase expression of miR-26a, miR-26a-1, and miR-122, as the suppressors in liver cancer, possibly resulting in a lower risk of developing liver cancer in female mice (Xie et al., 2017). Additionally, butyrate from microbiota induces apoptosis through up-regulation of miR-22 expression and repression of sirtuin1 (Sirt-1) expression in hepatic cells (Pant et al., 2017). FXR plays a pivotal role in host liver metabolism, including liver regeneration, hepatoprotection, prevention of NAFLD and hepatocarcinogenesis (Wang et al., 2008a,b; Chiang, 2013; Zhang et al., 2016). Zhang et al. (2016) reported that modulation of the gut microbiota by inhibition of intestinal FXR signaling 
alters host liver lipid metabolism and improves obesity-related metabolic dysfunction. Overall, FXR, miRNA, and SCFAs were relevant mediators of the gut's microbiota participation in liver disease.

\section{Microbiota and Gut-Lung Axis}

The study about gut-lung axis is in the initial stage, but it may potentially serve as a new direction for lung disease treatment (Budden et al., 2017). Asthma is a relatively stubborn bronchial disease. In childhood asthma, it may be closely related to decreasing relative abundance of the genus Faecalibacterium, Lachnospira, Veillonella, and Rothia and altered metabolites (Arrieta et al., 2015). Microbiota-accessible carbohydrates (particularly dietary fermentable fiber) can shape the lung immunity via changes in the microbiota and increase in SCFAs (Gray L.E. et al., 2017). Previous reports show that SCFAs may stimulate Tregs to protect against airway inflammation via activation of GPR43 (acetate and propionate) or inhibiting HDAC (butyrate) (Trompette et al., 2014; Thorburn et al., 2015; Gray L.E. et al., 2017). Dietary fermentable fiber leads to changes in the ratio of Firmicutes to Bacteroidetes and SCFA production. Propionate is capable of improving the bone marrow hematopoiesis of DC precursors. Furthermore, these DCs were in a position to stimulate $\mathrm{T}$ helper type 2 effector cells in the lung. The propionate-mediated mechanism may well protect lung from allergic airway inflammation and this process depends on GPR41 (Trompette et al., 2014). Trompette et al. (2014) found that high-fiber promoted the increase of acetate, leading to silencing transcription of Foxp3 genes in the lung, which were characterized by enhanced T-regulatory cells in numbers and function, and the process might lead to inhibition of allergic airways disease (a model for human asthma) (Thorburn et al., 2015). Gray J. et al. (2017) reported the interaction between the host immune system and the intestinal commensal bacteria. The colonization of gut commensal bacteria promotes the capability of IL-22-producing ILC3s (IL-22+ ILC3) in preferential trafficking to the lung, inducing IL-22 production and expression of the lung homing signal CCR4. Finally, it promotes the newborn's IL-22-dependent resistance to pneumonia (Gray J. et al., 2017; Tamburini and Clemente, 2017). In summary, T cell receptor signaling may be the primary pathway in the communication between gut and lung.

\section{Gut Microbiota-Bone Axis}

The gut microbiota is responsible for bone physiology, and it can regulate bone mass via the immune system and promote bone resorption and formation via SCFA production (Ohlsson and Sjogren, 2015; Yan et al., 2016). In detail, the increased bone mass in germ-free animals was associated with a reduction in inflammatory cytokine expression in bone and less osteoclastogenesis. The change of the gut microbiota composition caused by dietary changes, antibiotic treatments or pathogens induces the imbalance in metabolic and immune regulatory networks, affecting bone mass (Ohlsson and Sjogren, 2015). Probiotics and prebiotics, especially Lactobacillus and galactooligosaccharide, regulate bone metabolism and promote bone growth by altering the gut microbiota composition and maintaining or increasing mass (Britton et al., 2014; Ohlsson and Sjogren, 2015; Schwarzer et al., 2016). Lactobacillus may reduce the expression of two inflammatory cytokines, TNF- $\alpha$ and IL-1 $\beta$, increase the expression of osteoprotegerin (OPG), a potent inhibitor of osteoclastogenesis, and secrete beneficial immunomodulatory factors on ovariectomy-induced bone loss (Ohlsson et al., 2014). Galactooligosaccharide may improve calcium absorption and increase the relative proportion of bifidobacteria in the gut microbiota, possibly resulting in increasing bone mass (Whisner et al., 2013). The mechanisms involved the gut microbiota regulation of bone should be further investigated, since it might be a promising therapeutic theory for bone disease such as osteoporosis, osteoclastic bone resorption and rheumatoid arthritis.

\section{GUT MICROBIOTA-mIRNA INTERACTIONS IN PHYSIOLOGY AND DISEASE}

Dysregulation of specific miRNAs has been found in many diseases including breast cancer (Iorio et al., 2005; Chin et al., 2016), gastric cancer ( $\mathrm{Li}$ et al., 2015), non-small cell lung cancer (Yanaihara et al., 2006), and numerous inflammationrelated disease and cancers (Lujambio and Lowe, 2012). Both microbiota and miRNAs play key roles in health and disease. The gut microbiota-miRNA interactions comprise two processes: (i) host-secreted miRNAs regulate the gut microbiota; (ii) the gut microbiota affects the host via inducing special miRNAs (Belcheva, 2017). These physiological processes are linked to host health.

\section{Gut Microbiota-miRNA Interactions and Intestinal Epithelia}

The intestinal epithelial cells (IECs) are folded monolayer of cells that are exposed to the intestinal lumen (Gerbe et al., 2012). They play essential roles in nutrient absorption and hormone production, maintaining intestinal homeostasis (Peck et al., 2017). Moreover, when the microbiota communicates with other tissues, the intestinal epithelium is the first transmission channel for gut microbial signals (Schroeder and Backhed, 2016). Fecal miRNAs in gut lumen were partly from IEC. These miRNAs target bacterial mRNA, and then the host controls the gut microbiota via bacterial mRNA degradation or translational inhibition (Figure 1) (Liu et al., 2016). Liu et al. (2016) found that human miR-515-5p could target 16S rRNA/23S rRNA of Fusobacterium nucleatum and that miR1226-5p could affect the yegH gene of Escherichia coli (Liu et al., 2016). Also, Fusobacterium nucleatum and Escherichia coli have been previously found to drive CRC (Rubinstein et al., 2013; Liu et al., 2016). However, the mechanisms by which miRNAs enter bacteria and interfere with specific mRNA transcription are not clear. A recent report showed that miRNAs could possibly enter the bacteria within endocytosis in in vitro experiments (Zhao et al., 2017). Delivery of miR155/let7g not only altered the microbiota, but also influenced cardiovascular 


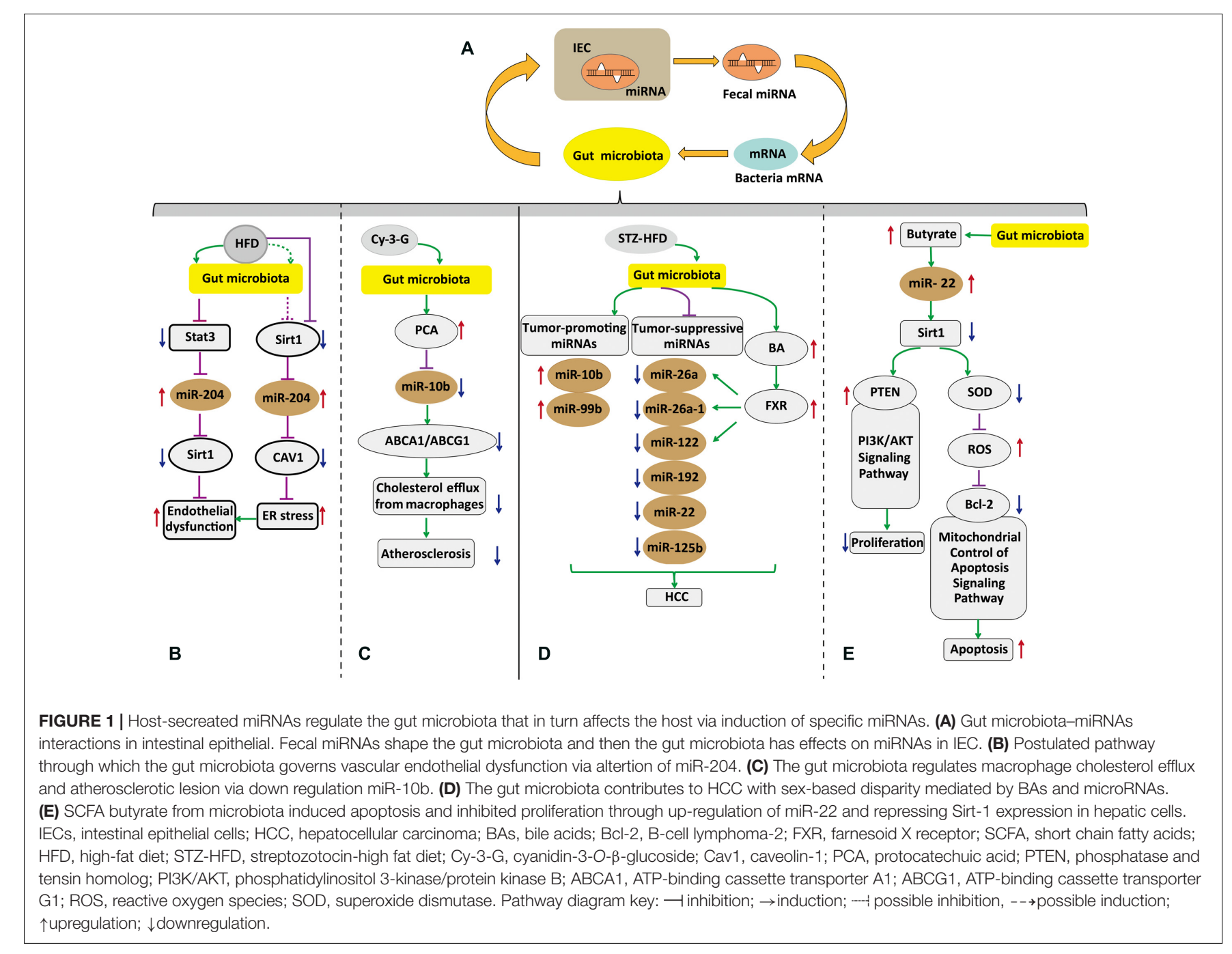

function (Zhao et al., 2017). Therefore, it could be believed that these intestinal miRNAs from IEC or other tissues might shape the gut microbiota and then induce dysbiosis. Peck et al. (2017) have confirmed that the microbiota regulates miRNA expression in IEC subtypes, and the regulation may alter intestinal homeostasis. Quantified miRNA analysis among germ-free, conventionalized and conventionally raised chowfed animals was achieved by miRquant. It was found that expression of some miRNAs are different among IEC subtypes and the difference depends on microbial status. Among these miRNAs, 19 miRNAs in intestinal epithelial stem cell (IESC) were significantly different. MiR-375-3p was the most activated, and only sensitive to the microbiota from IESC rather than other IEC subtypes. When miR-375-3p was knocked down, IESC proliferation increased (Peck et al., 2017). Similarly, Nakata et al. (2017) found that commensal bacteria induced the expression of miR-21-5p in IECs. However, there is no direct evidence showing how the microbiota regulates miRNAs. Possible mechanisms are based on the regulation of bacterial endotoxins and some metabolites or immune or mesenchymal cells signaling. As discussed above, fecal miRNAs from IEC affect bacterial gene expression, and the microbiota controls some miRNA expression in IESCs (Figure 1). These detailed mechanisms by which miRNAs participate in mediation of the gut microbiota and how microbiota affects miRNAs in IEC remain to be further investigated.

\section{Gut Microbiota-miRNA Interactions and Liver Disease}

The gut microbiota promotes or suppresses liver disease, where, part of the alterations were attributed to the interaction between microbiota-miRNAs. Xie et al. (2017) reported that the gut microbiota is implicated in bile acid metabolism and miRNA expression to induce HCC with sex-based disparity (Figure 1). Differential expression of bile acid synthesis, transport genes, and differential accumulation of hepatic bile acid between female and male mice resulted in a sex-dependent incidence of liver carcinogenesis (Xie et al., 2017). Also, these altered miRNAs could possibly interfere with other signaling pathways affecting homeostasis. Further studies are needed to explore the signaling molecules from the gut microbiota to miRNAs and bile acid, and may 
provide a novel perspective to treat HCC with sex-based disparity.

Butyrate produced by the microbiota in vivo is involved in numerous functions of host physiology, including tumor suppression. The mechanisms by which butyrate suppresses tumor progression are different depending on the type of cancer cells (Stilling et al., 2016; Bultman, 2017). Pant et al. (2017) reported that the role of microbiota-miRNAs was linked by butyrate in hepatic cells. They found that butyrate upregulated miR-22 expression, followed by SIRT-1 downregulation, resulting in hepatic cell apoptosis. Although the proposed mechanism needs to be confirmed in vivo, it supplied valuable information for HCC treatment.

\section{PROSPECTS}

The gut microbiota is emerging as a crucial keeper of host's health. Altered gut microbiota has been linked to various diseases. However the mechanisms by which the gut microbiota affects host's health are poorly known. For example, further in vivo studies need to be performed to confirm the function of the gut microbiota-mediated miR22 expression in liver cancer. Insight into the mechanisms of CNS diseases based on the microbiota gut-brain axis will provided a new research direction for studying CNS diseases. In addition, the gut microbiota, directly or indirectly, maintains homeostasis in many organ systems, but the mechanisms remain to be studied in detail. To conclude, the

\section{REFERENCES}

Arrieta, M. C., Stiemsma, L. T., Dimitriu, P. A., Thorson, L., Russell, S., Yuristdoutsch, S., et al. (2015). Early infancy microbial and metabolic alterations affect risk of childhood asthma. Sci. Transl. Med. 7:307ra152. doi: 10.1126/scitranslmed.aab2271

Bakker, A. D., Gakes, T., Hogervorst, J. M., De Wit, G. M., KleinNulend, J., and Jaspers, R. T. (2016). Mechanical stimulation and IGF-1 enhance mRNA translation rate in osteoblasts via activation of the AKT-mTOR pathway. J. Cell. Physiol. 231, 1283-1290. doi: 10.1002/jcp. 25228

Barrett, E., Ross, R. P., O’toole, P. W., Fitzgerald, G. F., and Stanton, C. (2012). $\gamma$-Aminobutyric acid production by culturable bacteria from the human intestine. J. Appl. Microbiol. 113, 411-417. doi: 10.1111/j.1365-2672.2012. 05344.x

Baruch, K., and Schwartz, M. (2016). Circulating monocytes in between the gut and the mind. Cell Stem Cell 18, 689-691. doi: 10.1016/j.stem.2016.05.008

Belcheva, A. (2017). MicroRNAs at the epicenter of intestinal homeostasis. Bioessays 39. doi: 10.1002/bies.201600200

Betrapally, N. S., Gillevet, P. M., and Bajaj, J. S. (2017). Gut microbiome and liver disease. Transl. Res. 179, 49-59. doi: 10.1016/j.trsl.2016.07.005

Britton, R. A., Irwin, R., Quach, D., Schaefer, L., Zhang, J., Lee, T., et al. (2014). Probiotic L. reuteri treatment prevents bone loss in a menopausal ovariectomized mouse model. J. Cell. Physiol. 229, 1822-1830. doi: 10.1002/jcp. 24636

Budden, K. F., Gellatly, S. L., Wood, D. L., Cooper, M. A., Morrison, M., Hugenholtz, P., et al. (2017). Emerging pathogenic links between microbiota and the gut-lung axis. Nat. Rev. Microbiol. 15, 55-63. doi: 10.1038/nrmicro. 2016.142

Bultman, S. J. (2017). Interplay between diet, gut microbiota, epigenetic events, and colorectal cancer. Mol. Nutr. Food Res. 61. doi: 10.1002/mnfr.201500902 gut microbiota has physiological and pathological functions. Further investigation of the physiological function of the gut microbiota may supply promising and effective treatments in complex diseases.

\section{AUTHOR CONTRIBUTIONS}

QF wrote the manuscript. Y-DW and W-DC edited and revised the manuscript.

\section{FUNDING}

This work was supported by the National Natural Science Foundation of China (Grant Nos. 81672433 and 81370537) to Y-DW, the National Natural Science Foundation of China (Grant Nos. 81472232 and 81270522), Program for Science and Technology Innovation Talents in Universities of Henan Province (HASTIT, Grant No. 13HASTIT024) and Plan for Scientific Innovation Talent of Henan Province to W-DC, and the Fundamental Research Funds for the Central Universities (Grant Nos. PYBZ1706 and PT1624) to Y-DW.

\section{ACKNOWLEDGMENTS}

We apologize to colleagues whose work could not be cited due to space limitations.

Buret, A. G. (2016). Good bugs, bad bugs in the gut: the role of microbiota dysbiosis in chronic gastrointestinal consequences of infection. Am. J. Gastroenterol. Suppl. 3, 25-32. doi: 10.1038/ajgsup.2016.11

Chiang, J. Y. (2013). Bile acid metabolism and signaling. Compr. Physiol. 3, 1191-1212. doi: 10.1002/cphy.c120023

Chin, A. R., Fong, M. Y., Somlo, G., Wu, J., Swiderski, P., Wu, X., et al. (2016). Cross-kingdom inhibition of breast cancer growth by plant miR159. Cell Res. 26, 217-228. doi: 10.1038/cr.2016.13

De Filippo, C., Cavalieri, D., Di Paola, M., Ramazzotti, M., Poullet, J. B., Massart, S., et al. (2010). Impact of diet in shaping gut microbiota revealed by a comparative study in children from Europe and rural Africa. Proc. Natl. Acad. Sci. U.S.A. 107, 14691-14696. doi: 10.1073/pnas.1005963107

Dinan, T. G., and Cryan, J. F. (2017). Gut-brain axis in 2016: brain-gut-microbiota axis - mood, metabolism and behaviour. Nat. Rev. Gastroenterol. Hepatol. 14, 69-70. doi: 10.1038/nrgastro.2016.200

Donohoe, D., Garge, N., Zhang, X., Sun, W., O'connell, T., Bunger, M., et al. (2011). The microbiome and butyrate regulate energy metabolism and autophagy in the mammalian colon. Cell Metab. 13, 517-526. doi: 10.1016/j.cmet.2011.02.018

Farrokhyar, F., Swarbrick, E. T., and Irvine, E. J. (2001). A critical review of epidemiological studies in inflammatory bowel disease. Scand. J. Gastroenterol. $36,2-15$.

Foster, J. A., and McVey Neufeld, K. A. (2013). Gut-brain axis: how the microbiome influences anxiety and depression. Trends Neurosci. 36, 305-312. doi: 10.1016/ j.tins.2013.01.005

Fung, K. Y., Cosgrove, L., Lockett, T., Head, R., and Topping, D. L. (2012). A review of the potential mechanisms for the lowering of colorectal oncogenesis by butyrate. Br. J. Nutr. 108, 820-831. doi: 10.1017/S00071145120 01948

Fung, T. C., Olson, C. A., and Hsiao, E. Y. (2017). Interactions between the microbiota, immune and nervous systems in health and disease. Nat. Neurosci. 20, 145-155. doi: $10.1038 / \mathrm{nn} .4476$ 
Gao, Z., Guo, B., Gao, R., Zhu, Q., and Qin, H. (2015). Microbiota disbiosis is associated with colorectal cancer. Front. Microbiol. 6:20. doi: 10.3389/fmicb. 2015.00020

Gerbe, F., Legraverend, C., and Jay, P. (2012). The intestinal epithelium tuft cells: specification and function. Cell Mol. Life Sci. 69, 2907-2917. doi: 10.1007/ s00018-012-0984-7

Gray, J., Oehrle, K., Worthen, G., Alenghat, T., Whitsett, J., and Deshmukh, H. (2017). Intestinal commensal bacteria mediate lung mucosal immunity and promote resistance of newborn mice to infection. Sci. Transl. Med. 9:eaaf9412. doi: 10.1126/scitranslmed.aaf9412

Gray, L. E., O'hely, M., Ranganathan, S., Sly, P. D., and Vuillermin, P. (2017). The maternal diet, gut bacteria, and bacterial metabolites during pregnancy influence offspring asthma. Front. Immunol. 8:365. doi: 10.3389/fimmu.2017. 00365

Holmes, E., Li, J. V., Athanasiou, T., Ashrafian, H., and Nicholson, J. K. (2011). Understanding the role of gut microbiome-host metabolic signal disruption in health and disease. Trends Microbiol. 19, 349-359. doi: 10.1016/j.tim.2011. 05.006

Iorio, M. V., Ferracin, M., Liu, C. G., Veronese, A., Spizzo, R., Sabbioni, S., et al. (2005). MicroRNA gene expression deregulation in human breast cancer. Cancer Res. 65, 7065-7070. doi: 10.1158/0008-5472.CAN-05-1783

Karbach, S. H., Schonfelder, T., Brandao, I., Wilms, E., Hormann, N., Jackel, S., et al. (2016). Gut microbiota promote angiotensin II-induced arterial hypertension and vascular dysfunction. J. Am. Heart Assoc. 5:e03698. doi: 10.1161/JAHA. 116.003698

Kennedy, P. J., Cryan, J. F., Dinan, T. G., and Clarke, G. (2017). Kynurenine pathway metabolism and the microbiota-gut-brain axis. Neuropharmacology 112, 399-412. doi: 10.1016/j.neuropharm.2016.07.002

Knights, D., Silverberg, M. S., Weersma, R. K., Gevers, D., Dijkstra, G., Huang, H., et al. (2014). Complex host genetics influence the microbiome in inflammatory bowel disease. Genome Med. 6:107. doi: 10.1186/s13073-014-0107-1

Köhler, C. A., Maes, M., Slyepchenko, A., Berk, M., Solmi, M., Lanctôt, K. L., et al. (2016). The gut-brain axis, including the microbiome, leaky gut and bacterial translocation: mechanisms and pathophysiological role in Alzheimer's disease. Curr. Pharmaceut. Design 22, 6152-6166. doi: 10.2174/ 1381612822666160907093807

Kostic, A. D., Chun, E., Robertson, L., Glickman, J. N., Gallini, C. A., Michaud, M., et al. (2013). Fusobacterium nucleatum potentiates intestinal tumorigenesis and modulates the tumor-immune microenvironment. Cell Host Microbe 14, 207-215. doi: 10.1016/j.chom.2013.07.007

Leue, C., Kruimel, J., Vrijens, D., Masclee, A., Van Os, J., and Van Koeveringe, G. (2017). Functional urological disorders: a sensitized defence response in the bladder-gut-brain axis. Nat. Rev. Urol. 14, 153-163. doi: 10.1038/nrurol. 2016.227

Li, P., Shan, J. X., Chen, X. H., Zhang, D., Su, L. P., Huang, X. Y., et al. (2015). Epigenetic silencing of microRNA-149 in cancer-associated fibroblasts mediates prostaglandin E2/interleukin-6 signaling in the tumor microenvironment. Cell Res. 25, 588-603. doi: 10.1038/cr.2015.51

Li, R., Yang, J., Saffari, A., Jacobs, J., Baek, K. I., Hough, G., et al. (2017). Ambient ultrafine particle ingestion alters gut microbiota in association with increased atherogenic lipid metabolites. Sci. Rep. 7:42906. doi: 10.1038/srep42906

Liu, S., Da Cunha, A. P., Rezende, R. M., Cialic, R., Wei, Z., Bry, L., et al. (2016). The host shapes the gut microbiota via fecal microRNA. Cell Host Microbe 19, 32-43. doi: 10.1016/j.chom.2015.12.005

Lujambio, A., and Lowe, S. W. (2012). The microcosmos of cancer. Nature 482, 347-355. doi: 10.1038/nature10888

Mu, C., Yang, Y., and Zhu, W. (2016). Gut microbiota: the brain peacekeeper. Front. Microbiol. 7:345. doi: 10.3389/fmicb.2016.00345

Nakata, K., Sugi, Y., Narabayashi, H., Kobayakawa, T., Nakanishi, Y., Tsuda, M., et al. (2017). Commensal microbiota-induced microRNA modulates intestinal epithelial permeability through a small GTPase ARF4. J. Biol. Chem. 292, 15426-15433. doi: 10.1074/jbc.M117.788596

Nicolas, S., Blasco-Baque, V., Fournel, A., Gilleron, J., Klopp, P., Waget, A., et al. (2017). Transfer of dysbiotic gut microbiota has beneficial effects on host liver metabolism. Mol. Syst. Biol. 13:921. doi: 10.15252/msb.201 67356

Ohigashi, S., Sudo, K., Kobayashi, D., Takahashi, O., Takahashi, T., Asahara, T., et al. (2013). Changes of the intestinal microbiota, short chain fatty acids, and fecal $\mathrm{pH}$ in patients with colorectal cancer. Dig. Dis. Sci. 58, 1717-1726. doi: 10.1007/s10620-012-2526-4

Ohlsson, C., Engdahl, C., Fak, F., Andersson, A., Windahl, S. H., Farman, H. H., et al. (2014). Probiotics protect mice from ovariectomy-induced cortical bone loss. PLoS One 9:e92368. doi: 10.1371/journal.pone.0092368

Ohlsson, C., and Sjogren, K. (2015). Effects of the gut microbiota on bone mass. Trends Endocrinol. Metab. 26, 69-74. doi: 10.1016/j.tem.2014.11.004

O’Keefe, S. J., Ou, J., Aufreiter, S., O'connor, D., Sharma, S., Sepulveda, J., et al. (2009). Products of the colonic microbiota mediate the effects of diet on colon cancer risk. J. Nutr. 139, 2044-2048. doi: 10.3945/jn.109.104380

Pant, K., Yadav, A. K., Gupta, P., Islam, R., Saraya, A., and Venugopal, S. K. (2017). Butyrate induces ROS-mediated apoptosis by modulating miR-22/SIRT1 pathway in hepatic cancer cells. Redox Biol. 12, 340-349. doi: 10.1016/j.redox. 2017.03.006

Patel, T., Bhattacharya, P., and Das, S. (2016). Gut microbiota: an indicator to gastrointestinal tract diseases. J. Gastrointest. Cancer 47, 232-238. doi: 10.1007/ s12029-016-9820-x

Peck, B. C., Mah, A. T., Pitman, W. A., Ding, S., Lund, P. K., and Sethupathy, P. (2017). Functional transcriptomics in diverse intestinal epithelial cell types reveals robust microRNA sensitivity in intestinal stem cells to microbial status. J. Biol. Chem. 292, 2586-2600. doi: 10.1074/jbc.M116.770099

Powell, N., Walker, M. M., and Talley, N. J. (2017). The mucosal immune system: master regulator of bidirectional gut-brain communications. Nat. Rev. Gastroenterol. Hepatol. 14, 143-159. doi: 10.1038/nrgastro.2016.191

Ridler, C. (2017). Bone: gut microbiota promote bone growth via IGF1. Nat. Rev. Endocrinol. 13:5. doi: 10.1038/nrendo.2016.200

Roy, S., and Trinchieri, G. (2017). Microbiota: a key orchestrator of cancer therapy. Nat. Rev. Cancer 17, 271-285. doi: 10.1038/nrc.2017.13

Rubinstein, M. R., Wang, X., Liu, W., Hao, Y., Cai, G., and Han, Y. W. (2013). Fusobacterium nucleatum promotes colorectal carcinogenesis by modulating E-cadherin/beta-catenin signaling via its FadA adhesin. Cell Host Microbe 14, 195-206. doi: 10.1016/j.chom.2013.07.012

Rupaimoole, R., and Slack, F. J. (2017). MicroRNA therapeutics: towards a new era for the management of cancer and other diseases. Nat. Rev. Drug Discov. 16, 203-222. doi: 10.1038/nrd.2016.246

Sampson, T. R., Debelius, J. W., Thron, T., Janssen, S., Shastri, G. G., Ilhan, Z. E., et al. (2016). Gut microbiota regulate motor deficits and neuroinflammation in a model of Parkinson's disease. Cell 167, 1469.e12-1480.e12. doi: 10.1016/j.cell. 2016.11.018

Sayin, S. I., Wahlstrom, A., Felin, J., Jantti, S., Marschall, H. U., Bamberg, K., et al. (2013). Gut microbiota regulates bile acid metabolism by reducing the levels of tauro-beta-muricholic acid, a naturally occurring FXR antagonist. Cell Metab. 17, 225-235. doi: 10.1016/j.cmet.2013.01.003

Schroeder, B. O., and Backhed, F. (2016). Signals from the gut microbiota to distant organs in physiology and disease. Nat. Med. 22, 1079-1089. doi: 10.1038/nm. 4185

Schwarzer, M., Makki, K., Storelli, G., Machuca-Gayet, I., Srutkova, D., Hermanova, P., et al. (2016). Lactobacillus plantarum strain maintains growth of infant mice during chronic undernutrition. Science 351, 854-857. doi: $10.1126 /$ science.aad 8588

Scott, D. W., Longpre, J. M., and Loo, G. (2008). Upregulation of GADD153 by butyrate: involvement of MAPK. DNA Cell Biol. 27, 607-614. doi: 10.1089/dna. 2008.0773

Shukla, S. D., Budden, K. F., Neal, R., and Hansbro, P. M. (2017). Microbiome effects on immunity, health and disease in the lung. Clin. Trans. Immunol. 6:e133. doi: 10.1038/cti.2017.6

Stempelj, M., Kedinger, M., Augenlicht, L., and Klampfer, L. (2007). Essential role of the JAK/STAT1 signaling pathway in the expression of inducible nitric-oxide synthase in intestinal epithelial cells and its regulation by butyrate. J. Biol. Chem. 282, 9797-9804. doi: 10.1074/jbc.M6094 26200

Stilling, R. M., Van De Wouw, M., Clarke, G., Stanton, C., Dinan, T. G., and Cryan, J. F. (2016). The neuropharmacology of butyrate: the bread and butter of the microbiota-gut-brain axis? Neurochem. Int. 99, 110-132. doi: 10.1016/j.neuint. 2016.06.011

Tamburini, S., and Clemente, J. C. (2017). Gut microbiota: neonatal gut microbiota induces lung immunity against pneumonia. Nat. Rev. Gastroenterol. Hepatol. 14, 263-264. doi: 10.1038/nrgastro.2017.34 
Thorburn, A. N., Mckenzie, C. I., Shen, S., Stanley, D., Macia, L., Mason, L. J., et al. (2015). Evidence that asthma is a developmental origin disease influenced by maternal diet and bacterial metabolites. Nat. Commun. 6:7320. doi: 10.1038/ ncomms 8320

Tremaroli, V., and Backhed, F. (2012). Functional interactions between the gut microbiota and host metabolism. Nature 489, 242-249. doi: 10.1038/ nature 11552

Trompette, A., Gollwitzer, E. S., Yadava, K., Sichelstiel, A. K., Sprenger, N., NgomBru, C., et al. (2014). Gut microbiota metabolism of dietary fiber influences allergic airway disease and hematopoiesis. Nat. Med. 20, 159-166. doi: 10.1038/ nm. 3444

Van de Wiele, T., Van Praet, J. T., Marzorati, M., Drennan, M. B., and Elewaut, D. (2016). How the microbiota shapes rheumatic diseases. Nat. Rev. Rheumatol. 12, 398-411. doi: 10.1038/nrrheum.2016.85

Villa, C. R., Ward, W. E., and Comelli, E. M. (2017). Gut microbiota-bone axis. Crit. Rev. Food Sci. Nutr. 57, 1664-1672. doi: 10.1080/10408398.2015.1010034

Wang, Y. D., Chen, W. D., Moore, D. D., and Huang, W. (2008a). FXR: a metabolic regulator and cell protector. Cell Res. 18, 1087-1095. doi: 10.1038/cr.200 8.289

Wang, Y. D., Chen, W. D., Wang, M., Yu, D., Forman, B. M., and Huang, W. (2008b). Farnesoid X receptor antagonizes nuclear factor kappaB in hepatic inflammatory response. Hepatology 48, 1632-1643. doi: 10.1002/hep. 22519

Whisner, C. M., Martin, B. R., Schoterman, M. H., Nakatsu, C. H., Mccabe, L. D., Mccabe, G. P., et al. (2013). Galacto-oligosaccharides increase calcium absorption and gut bifidobacteria in young girls: a double-blind cross-over trial. Br. J. Nutr. 110, 1292-1303. doi: 10.1017/S000711451300055X

Xie, G., Wang, X., Zhao, A., Yan, J., Chen, W., Jiang, R., et al. (2017). Sex-dependent effects on gut microbiota regulate hepatic carcinogenic outcomes. Sci. Rep. 7:45232. doi: $10.1038 /$ srep45232

Xue, L., He, J., Gao, N., Lu, X., Li, M., Wu, X., et al. (2017). Probiotics may delay the progression of nonalcoholic fatty liver disease by restoring the gut microbiota structure and improving intestinal endotoxemia. Sci. Rep. 7:45176. doi: $10.1038 /$ srep45176

Yan, J., Herzog, J. W., Tsang, K., Brennan, C. A., Bower, M. A., Garrett, W. S., et al. (2016). Gut microbiota induce IGF-1 and promote bone formation and growth. Proc. Natl. Acad. Sci. U.S.A. 113, E7554-E7563. doi: 10.1073/pnas.16072 35113
Yanaihara, N., Caplen, N., Bowman, E., Seike, M., Kumamoto, K., Yi, M., et al. (2006). Unique microRNA molecular profiles in lung cancer diagnosis and prognosis. Cancer Cell 9, 189-198. doi: 10.1016/j.ccr.2006.01.025

Yang, T., Owen, J. L., Lightfoot, Y. L., Kladde, M. P., and Mohamadzadeh, M. (2013). Microbiota impact on the epigenetic regulation of colorectal cancer. Trends Mol. Med. 19, 714-725. doi: 10.1016/j.molmed.2013.08.005

Zeng, H., Lazarova, D. L., and Bordonaro, M. (2014). Mechanisms linking dietary fiber, gut microbiota and colon cancer prevention. World J. Gastrointest Oncol. 6, 41-51. doi: 10.4251/wjgo.v6.i2.41

Zhang, L., Xie, C., Nichols, R. G., Chan, S. H., Jiang, C., Hao, R., et al. (2016). Farnesoid X Receptor Signaling Shapes the Gut Microbiota and Controls Hepatic Lipid Metabolism. mSystems 1:e00070-16. doi: 10.1128/mSystems. 00070-16

Zhang, Y., Zhou, L., Bao, Y. L., Wu, Y., Yu, C. L., Huang, Y. X., et al. (2010). Butyrate induces cell apoptosis through activation of JNK MAP kinase pathway in human colon cancer RKO cells. Chem. Biol. Interact 185, 174-181. doi: 10.1016/j.cbi.2010.03.035

Zhao, L., Zhou, X., Cai, W., Shi, R., Yang, G., and Yang, L. (2017). Host intestinal epithelium derived mirnas shape the microbiota and its implication in cardiovascular diseases. J. Am. College Cardiol. 69:1075. doi: 10.1016/S07351097(17)34464-9

Zhu, W., Gregory, J. C., Org, E., Buffa, J. A., Gupta, N., Wang, Z., et al. (2016). Gut Microbial Metabolite TMAO Enhances Platelet Hyperreactivity and Thrombosis Risk. Cell 165, 111-124. doi: 10.1016/j.cell.2016.02.011

Zhu, Y., Michelle Luo, T., Jobin, C., and Young, H. A. (2011). Gut microbiota and probiotics in colon tumorigenesis. Cancer Lett. 309, 119-127. doi: 10.1016/j. canlet.2011.06.004

Conflict of Interest Statement: The authors declare that the research was conducted in the absence of any commercial or financial relationships that could be construed as a potential conflict of interest.

Copyright (c) 2018 Feng, Chen and Wang. This is an open-access article distributed under the terms of the Creative Commons Attribution License (CC BY). The use, distribution or reproduction in other forums is permitted, provided the original author(s) and the copyright owner are credited and that the original publication in this journal is cited, in accordance with accepted academic practice. No use, distribution or reproduction is permitted which does not comply with these terms. 